\title{
Neuromechanics of Multifunctionality during Rejection in Aplysia californica
}

\author{
Hui Ye, ${ }^{1}$ Douglas W. Morton, ${ }^{2}$ and Hillel J. Chiel ${ }^{1,2,3}$ \\ Departments of ${ }^{1}$ Biomedical Engineering, ${ }^{2}$ Neurosciences, and ${ }^{3}$ Biology, Case Western Reserve University, Cleveland, Ohio 44106-7080
}

\begin{abstract}
How are the same muscles and neurons used to generate qualitatively different behaviors? We studied this question by analyzing the biomechanical and neural mechanisms of rejection responses in the marine mollusk Aplysia californica and compared these mechanisms with those used to generate swallowing responses (Ye et al., 2006). During rejection, the central grasper of the feeding structure closes to push inedible food out of the buccal cavity. This contrasts with swallowing, during which the grasper is open as it moves toward the jaws (protracts). We examined how the shape change of the grasper during rejection mechanically reconfigured the surrounding musculature. Grasper shape change increased the effectiveness of protractor muscle I2. The closed grasper alters the function of another muscle, the hinge, which becomes capable of inducing ventral rotations of rejected material. In contrast, during large-amplitude swallows, the hinge muscle mediates dorsal rotations of ingested material. Finally, after the grasper opens, its change in shape induces a delay in the activation of other surrounding muscles, the I1/I3/jaw complex, whose premature activation would close the halves of the grasper and induce it to pull inedible material back inward. The delay in activation of the I1/I3/jaw complex is partially attributable to identified multiaction neurons B4/B5. The results suggest that multifunctionality emerges from a periphery in which flexible coalitions of muscles may perform different functions in different mechanical contexts and in which neural circuitry is capable of reorganizing to exploit these coalitions by changes in phasing, duration, and intensity of motor neuronal activation.
\end{abstract}

Key words: Aplysia; biomechanics; multifunctionality; pattern generator; feeding; mechanical reconfiguration

\section{Introduction}

Multifunctional engineered devices combine single-function devices. For example, a Swiss Army knife can be used as a scissors, awl, or bottle opener by moving the correct tool into the active position and putting away all its other tools. In contrast, animals use the same peripheral structures for multiple functions. For example, the human hand may be used for punching, grasping, or playing piano, and the human tongue participates in talking, breathing, and feeding. What mechanisms underlie the multifunctionality of evolved systems?

A major source of behavioral multifunctionality is the flexibility of the nervous system. Studies of the neural architecture of the stomatogastric nervous system revealed that it dynamically reorganizes (Harris-Warrick et al., 1992; Weimann and Marder, 1994). Neurons may enter or leave motor patterns, and groups of neurons that generate independent patterns may join together to form new patterns. Evidence of reorganizing neural architectures

\footnotetext{
Received July 24, 2006; revised Sept. 13, 2006; accepted Sept. 14, 2006.

This work was supported by Whitehall Foundation Grant M97-17, National Science Foundation Grant IBN 0218386, and National Institutes of Health Grant NS047073. We thank Dr. Richard F. Drushel for assistance in illustrating the anatomy of the buccal mass and Dr. Gregory P. Sutton for comments on a previous draft of this manuscript.

Correspondence should be addressed to Dr. Hillel J.Chiel, Department of Biology, DeGrace Hall 304, Case Western Reserve University, 2080 Adelbert Road, Cleveland, 0H 44106-7080. E-mail: hjc@case.edu.

H. Ye's present address: Division of Cellular and Molecular Biology, Toronto Western Research Institute, Toronto, Ontario, Canada M5T 258.

D. W. Morton's present address: Premier Medical Imaging, 580 West College Avenue, Marquette, MI 49855. D0I:10.1523/JNEUROSCI.3143-06.2006

Copyright $\odot 2006$ Society for Neuroscience $\quad 0270-6474 / 06 / 2610743-13 \$ 15.00 / 0$
}

has also been observed in vertebrates. For example, circuitry that underlies struggling and swimming shares common neural elements, as does circuitry that underlies different forms of scratching (Robertson and Stein, 1988; Soffe, 1991; Berkowitz, 2001, 2002).

Another source of behavioral multifunctionality is the flexibility of the periphery. The many degrees of freedom of complex limbs allow them to reach the same endpoint in their workspace through many different routes. As limbs move, the net torques exerted by their muscles may change sign, and thus muscle function depends on mechanical context. Rather than ignore these complexities, the nervous system exploits them to generate qualitatively different behaviors (Buneo et al., 1997; Weiss and Flanders, 2004).

How do neural control and biomechanics interact to generate multifunctional behavior? Multifunctionality is difficult to study, because it is hard to simultaneously characterize biomechanics and neural control. Because we established many of the neural and biomechanical mechanisms that underlie swallowing responses of different amplitude in the marine mollusk Aplysia californica (Ye et al., 2006), we sought to determine how these properties changed during a qualitatively different behavior, rejection. Magnetic resonance (MR) imaging showed that the central grasper (radula/odontophore) changes shape from spherical when it is open to ellispsoidal when it is closed (Neustadter et al., 2002b; Novakovic et al., 2006). We hypothesized that grasper shape change may alter the shapes and positions of surrounding muscles, and this mechanical reconfiguration is crucial for the neural control of rejection (Novakovic et al., 2006). 
In this study, we show that mechanical reconfiguration and changes in neural control are both crucial for rejection. Grasper shape change increases the strength of protractor muscle I2 (the intrinsic muscle 2). The muscle around which the grasper rotates, known as the "hinge" (Sutton et al., 2004a), can induce ventral rotations attributable to the closure of the grasper during protraction. Grasper shape change also induces a delay in activating the muscle complex that moves the grasper back toward the esophagus (the I1/I3/jaw complex) to allow the grasper to remain open and release inedible material. We show that activity in neurons B4/B5 mediates the delay. These studies suggest that multifunctionality emerges from both biomechanics and neural control.

\section{Materials and Methods}

A detailed description of the techniques used in these studies was published recently (Ye et al., 2006). Details of intracellular recordings and extracellular recordings in vitro and in vivo are provided there. The following description will focus on the materials and methods that differ from those described in the previous study.

Aplysia californica (300-350 g) were obtained from Marinus Scientific (Garden Grove, CA). To study rejection responses, animals were induced to swallow polyethylene tubes (outer diameter, $1.27 \mathrm{~mm}$; inner diameter, $0.86 \mathrm{~mm}$; PE90; BD Biosciences, Sparks, MD). The tube was marked every $1.67 \mathrm{~mm}$ to measure its outward movement (Morton and Chiel, 1993a). After a variable number of swallows, animals would reject the tubes. As animals switch from swallowing to rejecting tubes, they sometimes produce intermediate patterns, in which a tube may move in and move out slightly (Morton and Chiel, 1993a). To ensure that we focused solely on rejection responses, we excluded intermediate patterns and rejection responses that occurred immediately after an intermediate pattern or a swallow. To accurately analyze tube movements during some rejections, a two-axis video system was used. Two digital video camcorders (ZR40 and ZR60; Canon, Tokyo, Japan) were mounted within a Styrofoam platform to be at right angles to one another, to be coplanar, and to have the same magnification. Based on these measurements, kinematic criteria were developed that allowed us to distinguish smallerand larger-amplitude rejections (see Results).

To analyze the effects of I 2 stimulation and shape change of the odontophore, the buccal mass was dissected out of an anesthetized animal and placed in a transparent glass chamber $(10 \times 8 \times 6 \mathrm{~cm})$ filled with artificial seawater. A mirror at an angle of $45^{\circ}$ was placed in front of the preparation so that both side and front views could be simultaneously recorded using a digital video camera. The buccal mass was suspended by extrinsic muscles E2, E3, and E4 (Chiel et al., 1986), and the buccal ganglion was removed. An extracellular suction electrode was placed on the stump of the radular nerve (RN) after the branches of the radular nerve leading to the odontophore had been severed to stimulate the very thin I2 nerve. This nerve contains the entire pool of motor neurons for I2 (B31/B32, B61/B62), and thus stimulating this nerve is a good way to maximally excite the I2 muscle (Hurwitz et al., 1996). During the protraction phase of rejection, the tube is firmly held between the halves of the radula and moves rigidly with it. To study tube movements during the protraction phase of rejection, we therefore glued a polyethylene tube to the base of the radular cleft. To determine the effects of the shape of the radula/odontophore on the ability of $\mathrm{I} 2$ to protract, the halves of the radula/odontophore were initially moved to an open position (by gently pushing the radular stalk upward), they were then closed and held shut with glue, then the halves were gently separated, and the radula/odontophore was again opened.

The hinge, i.e., the muscle formed by the interdigitation of the base of the radula/odontophore and the surrounding muscles (Sutton et al., 2004a), could play a role in inducing rotation of a polyethylene tube during the protraction phase of strong rejections. To analyze the role of the hinge muscle on tube rotation during rejections, we gently pulled the radula/odontophore through the jaws and cut through the hinge muscle (Sutton et al., 2004a, their Fig. 1 B) at the base of the odontophore so that the odontophore was completely disconnected from the rest of the buccal mass. We then glued a tube onto the radular cleft, glued the radular halves together, and gently pushed the radula/odontophore back through the jaws. An extracellular stimulation electrode was attached to the I2 nerve as described above. Tube movements in response to electrical stimulation of the I 2 nerve were recorded using digital video.

To study the importance of the timing of the closure of the I1/I3/jaw complex relative to the protraction phase of small- or large-amplitude rejections, the buccal mass was dissected out of anesthetized animals, and extracellular electrodes were applied to the esophageal nerve, buccal nerve 2 (BN2), the main branch of the RN, and the I2 muscle. The buccal mass was suspended by extrinsic muscles E2, E3, and E4. A tube was placed between the radular halves. We induced egestive patterns by stimulating the esophageal nerve ( $20 \mathrm{~Hz}$ for $2 \mathrm{~s}$ with $1 \mathrm{~ms}$ pulses) (Weiss et al., 1986). At the cessation of I2 activity during an egestive pattern, we observed the onset of activity in BN2, which contains the axons of the majority of the motor neurons for the I1/I3/jaw complex, and recorded the net movement of the tube. In some preparations, we stimulated BN2 before $\mathrm{I} 2$ activity ceased, inducing the I1/I3/jaw complex to contract sooner than it would have naturally, and recorded the effects of this stimulation on tube movement.

\section{Results}

Predicted changes attributable to mechanical reconfiguration After Aplysia ingest inedible material, they reject it. The goal of an effective rejection is to use the central grasper (radula/odontophore) within the buccal mass to push inedible material out of the buccal cavity, so that the animal can find and ingest edible material (Kupfermann, 1974; Katzoff et al., 2006). The grasper first closes on the inedible material and moves it toward the outside world (protracts it), so that the inedible material is pushed out of the buccal cavity. The grasper then opens to release the inedible material, and the grasper moves inward toward the esophagus (retracts), leaving the inedible material behind (Kupfermann, 1974; Morton and Chiel, 1993a). Rejection primarily differs from ingestive behaviors (i.e., biting or swallowing) in the timing of the closure of the grasper. During ingestive behaviors (biting and swallowing), the grasper is open as it protracts and closes as it retracts, whereas during rejection, the phase relationship reverses: the grasper is now closed as it protracts and opens as it retracts (Morton and Chiel, 1993a). When open, the grasper assumes an approximately spherical shape, whereas when the grasper is closed, it assumes an ellipsoidal shape (Neustadter et al., 2002b; Novakovic et al., 2006).

How does grasper shape change alter the mechanics and neural control of other buccal muscles during rejection? We explored this question using kinetic models of the buccal mass based on anatomical studies and in vivo views of the buccal mass in intact, behaving animals obtained from magnetic resonance imaging (Sutton et al., 2004b; Novakovic et al., 2006). In the model, a central structure represented the change of the grasper from the opened to the closed state by changing its shape from spherical to ellipsoidal. Using the model, it was possible to make three predictions about how the shape change of the grasper would alter the function of specific muscles.

First, the MR images and the kinetic model predicted that, at the onset of rejection, the closing of the central grasper (inducing it to assume an elongated shape) would "mechanically reconfigure" the I2 protractor muscle by lengthening it, and this in turn would strengthen the ability of I 2 to generate protractive forces (Novakovic et al., 2006, their Figs. 2, 4).

Second, the MR images and the kinetic model predicted that, in response to strong protractions, the entire grasper (radula/ odontophore) would rotate about the hinge muscle, which could induce a rotation in the inedible material as that material was 
rejected (Novakovic et al., 2006, their Fig. 2C), thus creating a new behavioral effect for the hinge muscle.

Third, the MR images and the kinetic model predicted that the retraction phase of rejection would be altered by the change in the grasper shape (Novakovic et al., 2006, their Fig. 2). The grasper must open after the peak of retraction to release the inedible material. Closing the I1/I3/jaw complex around the grasper would tend to close the grasper and induce it to pull the rejected material back into the buccal cavity. As a consequence, to prevent "functional antagonism," the onset of activity in I1/I3 should be delayed, and retraction should be mediated by the hinge muscle.

In the following sections, we test these predictions of the effects of grasper shape change using in vitro preparations (see Figs. $1-3)$. We then test the hypothesis that the delay in onset of activity in the I1/I3/jaw complex may be attributable to activity in identified neurons B4/B5 (see Fig. 4). Finally, we test these neuromechanical predictions in vivo by examining rejection behavior (see Fig. 5) and neuromuscular activity (see Figs. 6-8) in intact, behaving animals, documenting several neural mechanisms for the differential deployment of the degrees of freedom of the buccal mass that are responsible for multifunctionality.

\section{Elongation of $\mathrm{I} 2$ by the closed radula/odontophore enhances the ability of $\mathrm{I} 2$ to protract}

The $\mathrm{I} 2$ muscle acts as a protractor and receives activation during biting, swallowing, and rejection (Hurwitz et al., 1996). During rejection, animals generate strong protractions (Morton and Chiel, 1993a), and I2 receives strong activation (Hurwitz et al., 1996, their Fig. 13). The strong protractions required for effective rejection pose a biomechanical problem for the $\mathrm{I} 2$ muscle. Previous studies have demonstrated that, during both swallowing and biting, the I2 muscle becomes increasingly less effective at mediating protraction as the radula/odontophore moves anteriorly. First, I2 loses mechanical advantage as the radula/odontophore moves anteriorly (Sutton et al., 2004b). Second, I2 shortens, and, previous studies of its length/tension properties indicate that, at short lengths, I2 can exert little force (Yu et al., 1999). Third, large anterior movements of the radula/odontophore stretch the hinge muscle, whose passive forces antagonize I2 (Sutton et al., 2004a). Given these constraints on the ability of I2 to exert force, how can I2 generate the very strong protractions observed during rejection?

Previous studies have demonstrated that closure of the radular halves elongates the radula/odontophore and causes it to assume an ellipsoidal shape (Neustadter et al., 2002a,b). Because the radula closes on inedible material at the beginning of rejection (Novakovic et al., 2006), the change in shape of the radula/ odontophore could stretch the thin I 2 muscle that surrounds the radula/odontophore, increasing both the mechanical advantage of $\mathrm{I} 2$ and its ability to generate force. Because previous studies have shown that $\mathrm{I} 2$ is more strongly activated during the protraction phase of rejection than it is during swallowing or biting (Hurwitz et al., 1996, their Fig. 13), an alternative explanation for the stronger protraction mediated by $\mathrm{I} 2$ during rejection would be that $\mathrm{I} 2$ is more strongly activated. To test the prediction of the effects of mechanical reconfiguration and to examine the relative contributions of mechanical reconfiguration and increased activation of the I2 muscle, we examined the effects of stimulating I2 on protraction intensity and tube movement when the halves of the radula were closed or open as I 2 was stimulated at different durations and frequencies (Fig. 1).

We varied the duration and frequency of stimulation of the I2 nerve, which contains all of the motor neurons for the I2 protractor muscle, placed the central grasper in an open or closed con- figuration, and examined the outward movement of a tube placed between the halves of the radula and glued to the base of the radular cleft (see Materials and Methods). When the halves of the radula were open, increasing the duration of I2 stimulation from 3 to $4 \mathrm{~s}$ while also increasing the frequency of stimulation from 10 to $20 \mathrm{~Hz}$ led to significantly larger outward tube movements $(2.1 \pm 0.6$ vs $5.0 \pm 0.8 \mathrm{~mm} ; n=4)$ (Fig. 1 , compare $A, B)$. Closing the halves of the radula and applying the lower stimulation protocol $(3 \mathrm{~s}$ at $10 \mathrm{~Hz})$ also generated larger protractions than were observed when the halves of the grasper were open $(5.0 \pm 0.8 \mathrm{~mm} ; n=4)$ (Fig. 1 , compare $A, C)$. Combining the two effects (closed radular halves, higher duration, and frequency of stimulation) led to an even larger outward translation of the tube, followed by a dorsal rotation of the tube. The outward translation was $10.0 \pm 1.6 \mathrm{~mm}$, and the dorsal rotation was $39 \pm 8^{\circ}[n=4$ (Fig. 1, compare $B, D$ ); overall multivariate ANOVA (MANOVA) was highly significant ( $p<0.006 ; n=4)$, and all comparisons between different groups were significant, except the comparison of $B$ and $C]$.

The results demonstrate that outward movements and rotations of a tube in response to 12 stimulation are enhanced when the halves of the radula are closed. In turn, these results suggest that increased neural activation of $\mathrm{I} 2$, in combination with mechanical reconfiguration attributable to a change in grasper shape, alters the effectiveness of I2 as a protractor muscle, as predicted by a previous study (Novakovic et al., 2006).

\section{Strong protractions induce rotations of the central grasper about the hinge muscle}

The change in grasper shape could also affect the function of another muscle, the hinge. Both the in vivo MR images (Novakovic et al., 2006) and the results obtained from long-duration, high-frequency stimulation of the I 2 protractor muscle suggested that, during strong protractions, the closed grasper could rotate about the hinge muscle (Sutton et al., 2004a).

We tested this hypothesis by examining the movement of a tube held by a closed radula in response to high-frequency, longduration stimulation of the I2 protractor muscle $(4 \mathrm{~s}$ at $20 \mathrm{~Hz}$ ) with and without the hinge muscle intact. Lesions of the hinge muscle had no effect on the amount of outward translation of the tube held by the grasper. In contrast, as predicted, lesions of the hinge muscle abolished the ventral rotation of the tube (Fig. 2). These results suggest that mechanical reconfiguration leads to a new behavioral function for the hinge muscle, the ability to induce ventral rotations of material held by the grasper.

\section{Delayed activation of the I1/I3/jaw complex is necessary for tube release}

After an animal has pushed inedible material out of the buccal cavity by closing and protracting the central grasper, the animal opens the grasper and releases the inedible material (Morton and Chiel, 1993a). What are the implications of this change in grasper shape for buccal mass function? If the grasper were to be forced closed near the peak of protraction, it would pull the inedible material back into the buccal cavity. Because the grasper is within the lumen of the I1/I3/jaw complex, premature contraction of the I1/I3/jaw complex is likely to close the grasper on the tube and disrupt rejection movements.

To test this hypothesis, we examined the effect of prematurely activating the I1/I3/jaw complex during small- and largeamplitude rejection-like movements induced by shocking the esophageal nerve (Weiss et al., 1986). By monitoring activity in the $\mathrm{I} 2$ muscle, we could determine when protraction activation 
ended and prematurely activate all of the motor neurons for the I1/I3/jaw complex by shocking BN2, through which the axons of the motor neurons for I1/I3 travel (Scott et al., 1991; Church and Lloyd, 1994). Based on the results of activating the $\mathrm{I} 2$ muscle (Fig. 1), we defined small-amplitude rejections as those in which a tube translated outward but did not rotate (Fig. 3A1) and large-amplitude rejections as those in which a tube both translated and rotated outward (Fig. 3B1).

We observed that, for both small- and large-amplitude rejections, premature activation of the I1/I3/jaw complex significantly reduced the net outward movement of the tube. For the smaller-amplitude rejections, the net outward movement was reduced from $6.0 \pm 1.6$ to $3.8 \pm 0.5 \mathrm{~mm}(p<$ $0.05 ; n=4$ ) (Fig. 3, compare A1, A2). For the larger-amplitude rejection, the net outward movement was reduced from $8.3 \pm$ 1.3 to $4.8 \pm 0.5 \mathrm{~mm}(p<0.01 ; n=4)$ (Fig. 3 , compare $B 1, B 2)$. No sign of noxious withdrawal was seen in response to these shocks, suggesting that their primary effect was to activate the motor neurons controlling the I1/I3/jaw complex. These results support the hypothesis that the change in shape of the grasper at the peak of protraction as it opens requires a delay in the onset of activity in the I1/I3/jaw complex. The delay ensures that the contraction of the I1/I3/ jaw complex does not force the grasper closed and prevent the release of inedible material. In turn, this implies that retraction cannot be initiated by activation of the I1/I3/jaw complex but must be initiated by activation of the hinge muscle, which has been shown to perform this function in large-amplitude swallows (Ye et al., 2006).

\section{B4/B5 neurons delay onset of activity in the I1/I3/jaw complex}

Mechanical reconfiguration attributable to the opening of the central grasper requires a delay in the onset of activity of the I1/I3/jaw complex. What neural mechanisms could delay the onset of I1/I3/jaw complex during rejection? Several lines of evidence suggest that the multiaction B4/B5 neurons may mediate this delay. First, the B4/B5 neurons make extensive inhibitory connections to the motor neurons of the I1/I3/jaw complex (e.g., B3, B6, and B10) (Gardner, 1971a,b). Second, the B4/B5 neurons are active at the relevant time to affect these motor neurons, because they are maximally active at the end of the protraction phase and the onset of the retraction phase (Morton and Chiel, 1993b, their Fig. 9). Third, extracellular recordings above the soma of B4/B5 demonstrated that activity in these

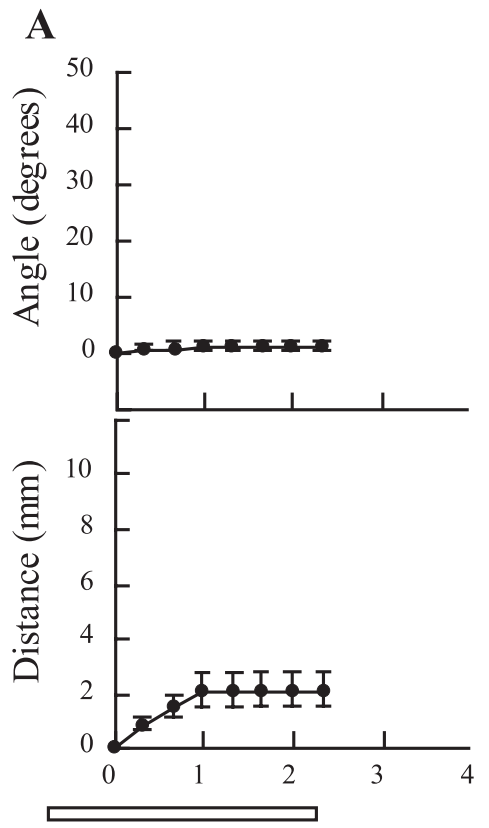

B

$10 \mathrm{~Hz}, 3 \mathrm{~s}$ I 2 stimulation $20 \mathrm{~Hz}, 4 \mathrm{~s}$ I 2 stimulation

Time (s)

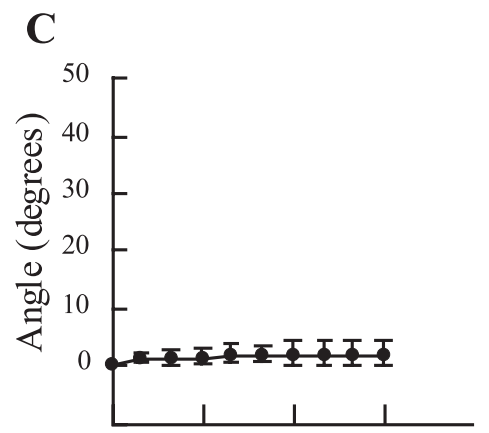

D
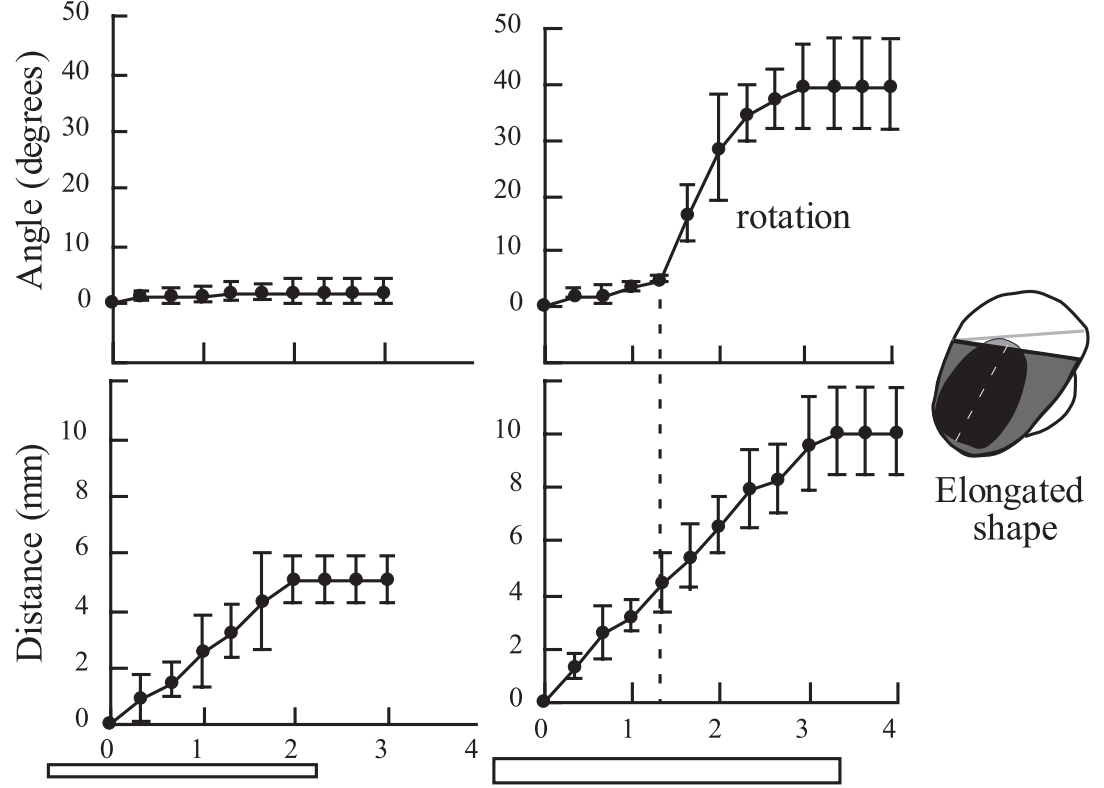

$10 \mathrm{~Hz}, 3 \mathrm{~s}$ I2 stimulation $20 \mathrm{~Hz}, 4 \mathrm{~s} \mathrm{I} 2$ stimulation

Time (s)

Figure 1. Closed odontophore and longer-duration, higher-frequency 12 stimulation can induce large-amplitude rejections. Insets to the right show schematic lateral views of the radula and odontophore, based on in vitro video measurements. Several anatomical structures are labeled (radular surface, base of radular cleft, radular stalk, prow, and odontophore) (for more details of the buccal mass anatomy, see Neustadter 2002a,b). $\boldsymbol{A}$, The 12 nerve was stimulated for $3 \mathrm{~s}$ at $10 \mathrm{~Hz}$. The halves of the radula were open, creating a radula/odontophore with a spherical shape (shown schematically to the right of the plots). The tube moved out a net distance of $2.1 \pm 0.6 \mathrm{~mm}$ (bottom) and showed no significant rotation (top). $\boldsymbol{B}$, Increasing the frequency and duration of $\mathrm{I} 2$ stimulation to $20 \mathrm{~Hz}$ for 4 s led to a much larger outward movement of the tube $(5.0 \pm 0.8 \mathrm{~mm})$ (bottom) but no rotation (top). C, Closing the halves of the radula, creating an elongated radula/odontophore (shown schematically to the right of the plots), increased the effect of stimulation 12 at $10 \mathrm{~Hz}$ for $3 \mathrm{~s}$ (total outward movement of the tube is $5.0 \pm 0.8 \mathrm{~mm}$ ) (bottom; compare with bottom of $\boldsymbol{A}$ ) but did not lead to any rotation of the tube (top). $\boldsymbol{D}$, (losing the halves of the radula, creating an elongated radula/odontophore, and stimulating $\mathrm{I} 2$ at a higher frequency for a longer duration $(20 \mathrm{~Hz}$ for $4 \mathrm{~s})$ induced an outward translation of a tube $(10.0 \pm 1.6 \mathrm{~mm})$ (bottom) and a rotation of the tube $\left(39 \pm 8^{\circ}\right)$. The initial outward movement of the tube began $0.6 \pm 0.2 \mathrm{~s}$ after the onset of $\mathrm{I} 2$ stimulation, and the rotation of the tube began $1.9 \pm 0.2 \mathrm{~s}$ after the onset of $\mathrm{I} 2$ stimulation. Overall MANOVA was highly significant ( $p<0.006 ; n=4$; and all comparisons between different parts of the figure were significant, except the comparison of $\boldsymbol{B}$ and $\boldsymbol{C})$. 
A

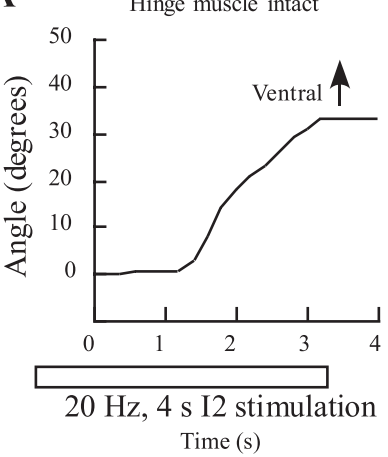

B

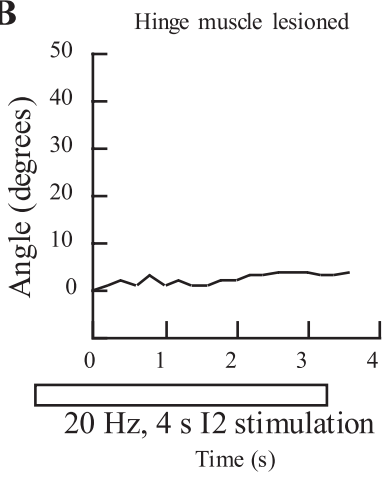

Figure 2. Lesioning the hinge muscle blocks the ventral rotation of the grasper and rejected material. $A$, The $\mathrm{I} 2$ protractor muscle was stimulated at a frequency of $20 \mathrm{~Hz}$ for $4 \mathrm{~s}$ in an isolated suspended buccal mass whose radular halves were closed around a tube. The tube moved outward and rotated ventrally. $\boldsymbol{B}$, The experiment was repeated after the hinge muscle was lesioned. Although the tube moved outward, it no longer rotated at all. The amount of outward movement before the lesion was $10.0 \pm 1.6 \mathrm{~mm}$ and after the lesion was $10.3 \pm 1.0 \mathrm{~mm}$, so the hinge muscle lesion only affected the ventral rotation of the tube $(n=3)$.

neurons occurs with high frequency during rejection behavior in intact, behaving animals (Warman and Chiel, 1995, their Fig. $8 B)$.

To test the hypothesis that the B4/B5 neurons could affect the timing of the onset of activity in the I1/I3/jaw muscle complex, we induced rejection-like patterns using esophageal nerve stimulation and then examined the effect of depolarizing or hyperpolarizing a single B4/B5 neuron on the delay from the end of protraction (the end of activity in the I2 muscle) to the onset of activity in the I1/I3/jaw complex motor neurons (the onset of large unit extracellular activity on BN2). Because of the high variability in rejection patterns (Church and Lloyd, 1994; Brezina et al., 2006), we compared the ratio of the delay of successive patterns, with and without altering the activity in a B4/B5 neuron. We found that increasing the activity of a single B4/B5 neuron by depolarizing it significantly delayed the onset of activity in motor neurons for the I1/I3/jaw complex. The delay to onset of activity in the I1/I3/jaw motor neurons was increased by $26 \pm 10 \%$ relative to the delay in the immediately preceding motor pattern $(p<$ $0.001 ; n=5$ ) (Fig. $4 A, B, D$ ). In contrast, decreasing the activity of a single B4/B5 neuron by hyperpolarizing it significantly reduced the delay to onset of activity in motor neurons for the I1/I3/jaw complex. The delay was decreased by $28 \pm 4 \%$ relative to the delay in the immediately preceding motor pattern $(p<0.001$; $n=4$ ) (Fig. $4 A, C, D$ ). These data suggest that changes in the activity of the B4/B5 neurons contribute to the delay to onset of activity in the I1/I3/jaw complex.

\section{Conditional role of hinge muscle predicted in vitro is observed in vivo}

The in vitro studies (Fig. 1) demonstrated that the mechanical reconfiguration of the I2 muscle attributable to the change in the shape of the central grasper at the onset of the protraction phase of rejection could account for the ability of I2 to generate strong protractions. Moreover, it suggested that the role of the hinge muscle could be altered by the new mechanical configuration. In a previous study, we demonstrated that, after it was stretched, the hinge muscle could generate dorsal rotations of a tube during large-amplitude (type B) swallows (Ye et al., 2006, their Fig. 7B). The data shown in Figures 1 and 2 suggest that, if the grasper is closed and I 2 is strongly activated, the hinge muscle could be

responsible for generating ventral rotations of a tube during large-amplitude rejections.

To test this hypothesis, we carefully measured the kinematics of tube rejection in intact, behaving animals. All rejections began with an outward translation of the tube. In some rejections, after the outward translation, the tube rotated ventrally within the midsagittal plane through the jaws of the animal (Fig. 5, compare $A, B)$. We plotted histograms of the maximum ventral angle generated by tubes during rejections and observed that one group of rejections showed no rotations, whereas another group showed rotations ranging from 13 to $85^{\circ}$. Thus, we classified any rejection in which a ventral rotation of $>7^{\circ}$ occurred as a type $\mathrm{B}$ rejection and all other rejections as type $\mathrm{A}$ rejections. Of the 85 rejections analyzed from 14 animals, 67\% (57 of 85 ) were type A and 33\% (28 of 85 ) were type B. Type B rejections were associated with a significantly larger outward movement of the tube than type A rejections (4.4 $\pm 0.8 \mathrm{~mm}$ during type A rejections vs $6.1 \pm 1.0$ $\mathrm{mm}$ during type $\mathrm{B}$ rejections; $p<0.001 ; n=85$ ). We also more frequently saw the surface of the radula during type $B$ than type $A$ rejections [ $35 \%$ of type A rejections ( 20 of 57 type A rejections) vs $54 \%$ type B rejections ( 15 of 28 type B rejections)], suggesting that type $B$ rejections were associated with larger-amplitude protractions. The amplitude of a rejection did not appear to affect the amplitude of a succeeding rejection. We used 34 sequences from 14 animals to construct a $2 \times 2$ contingency table (i.e., type $\mathrm{A}$ followed by type A, type A followed by type B, type B followed by type A, or type B followed by type B swallows). The $\chi^{2}$ statistic was not significant $(0.69 ; p>0.05)$. These results suggest that each rejection could be analyzed as an independent response.

The in vitro studies reported above (Fig. $1 D$ ) and the in vivo MR images (Novakovic et al., 2006, their Fig. 2) both suggested that the initial translation phase of both small- and largeamplitude rejections would be identical. To test this hypothesis, we used the two-axis video views to reconstruct the exact path of a mark on the tube as the animal rejected it in small- and largeamplitude rejections (details of reconstruction in Fig. $5 C$ ). We observed that the initial phases of both types of rejection were identical, consisting solely of an outward translation. In the larger-amplitude rejections, rotations of the tube were only observed after the initial translation phase was completed (Fig. 5D).

The in vitro studies (Fig. 1D) also suggested that the mechanical reconfiguration of the grasper that induced stronger protractions in $\mathrm{I} 2$ and rotation about the hinge muscle would contribute to the final total outward movement of the tube. To quantify this, we reconstructed and averaged six larger-amplitude rejections from four animals. We observed that rotation began $\sim 40 \%$ through the cycle (Fig. 5E) and was associated with a steady increase in the outward movement of the tube (Fig. $5 F$ ), as predicted by the in vitro study (Fig. 1D).

\section{In vitro predictions of effects of mechanical reconfiguration on neural control are observed in vivo}

Previous work and the studies reported above lead to testable predictions for how mechanical reconfiguration affects in vivo neural activity during rejection.

(1) Longer duration activity in the I2 protractor muscle should generate larger-amplitude protractions that involve not only outward translation of the tube but also ventral rotation (Figs. 1, 2) (Novakovic et al., 2006).

(2) Neural activity in the motor neuron for the hinge muscle, B7 (Ye et al., 2006), should be observed as the tube rotates ventrally. Furthermore, the onset of retraction should be associated with activity in the hinge muscle motor neuron B7 (Ye et al., 
2006), because this is the only muscle in this mechanical context that can induce a rotation that will initiate retraction (the grasper remains open, so it cannot contribute to retraction as it does in swallowing) (Ye et al., 2006).

(3) For the open grasper to release inedible material, it should not be compressed by the I1/I3/jaw complex (Fig. 3). As a consequence, the onset of activity in motor neurons for I1/I3 should be delayed, and this delay should be greater in the larger-amplitude (type B) rejections, because they involve larger protractions.

(4) The delay in onset of activity in the I1/I3/jaw complex should be associated with an increase in the activity of the B4/B5 neurons, which may act directly to delay activity in these motor neurons (Fig. 4). Furthermore, activity in B4/B5 should be greater in larger-amplitude rejections, because the larger-amplitude protraction implies a longer delay until the radula returns to a position in which activating the I1/I3/jaw complex will not induce it to close on inedible material and pull the material back into the buccal cavity (Fig. 3).

Ideally, one would want to test these predictions directly by recording from individual identified neurons in intact, behaving animals while altering their activity, or the shape of the central grasper, at different times throughout a rejection response. Because this is not yet technically feasible, we did a correlative study of these predictions by recording from relevant nerves and muscles in intact, behaving animals. Previous work has demonstrated that it is possible to track the activity of groups of identified motor neurons by recording from appropriate nerves and muscles in Aplysia. In particular, electromyogram (EMG) activity recorded from the I2 protractor muscle monitors activity in the motor neurons of I2 (B31, B32, B61, and B62) (Hurwitz et al., 1996). Activity of the motor neurons (B8a, B8b) that innervate the major muscle (I4) that induces the grasper to close (Morton and Chiel, 1993b) appears as large unit extracellular activity on the RN (Morton and Chiel, 1993a,b). The posterior part of the I1/I3/jaw complex is innervated by the B10 motor neurons (Church and Lloyd, 1994), which appear among the third largest extracellular units on BN2 (Morton and Chiel, 1993b). Other motor neurons that innervate the I1/I3/jaw complex (e.g., B3 and B6) (Church and Lloyd, 1994) appear among the second and first largest units on BN3 (Scott et al., 1991). The level of activity in the multiaction B4/B5 neurons can be monitored by recording the largest extracellular unit on BN3 (Warman and Chiel, 1995). Activity in the B7 motor neurons, which innervate the hinge muscle, can be monitored by recording the third largest unit on BN3 (Ye et al., 2006, their Fig. 6). Thus, by implanting extracellular recording electrodes on the $\mathrm{I} 2$ muscle, or $\mathrm{RN}, \mathrm{BN} 2$, and $\mathrm{BN} 3$, and using a

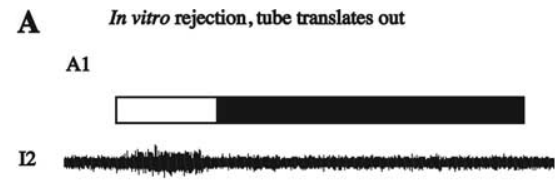

A2
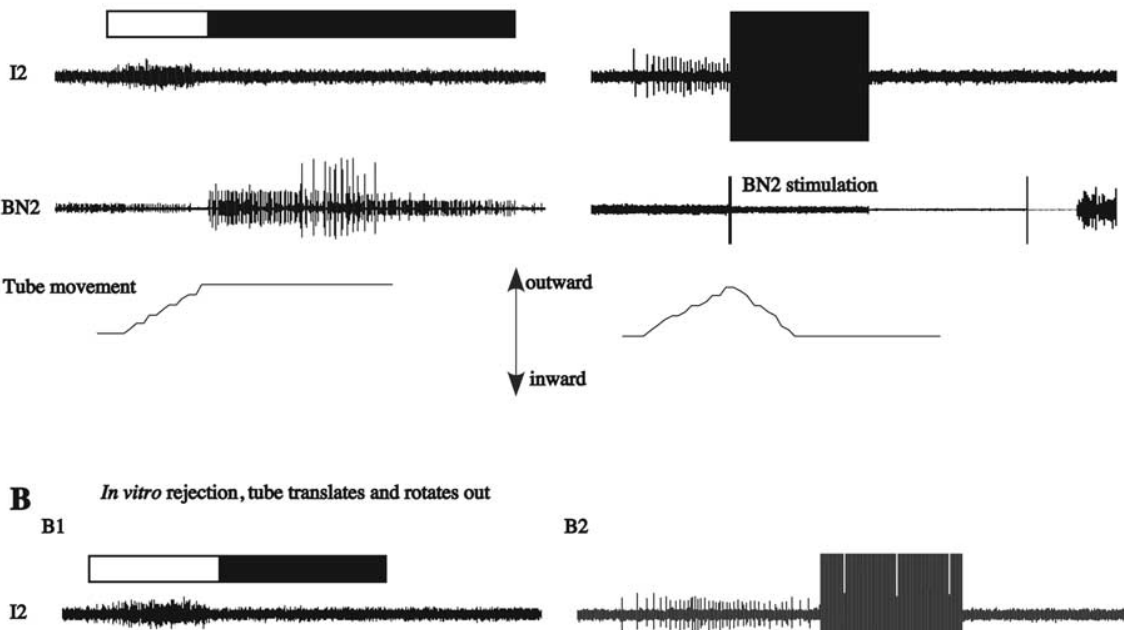

B2

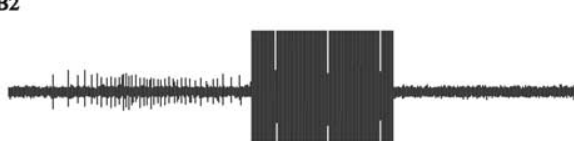

Figure 3. Activating the $11 / 13 /$ jaw complex prematurely during in vitro egestion-like patterns reduces the net outward movement of a tube. $\boldsymbol{A}$, Premature activation of the I1/I3/jaw complex during a smaller-amplitude rejection-like pattern. $\boldsymbol{A} \mathbf{1}, \mathrm{A}$ Open bar above the traces indicates the duration of the protraction phase, based on 12 EMG. Filled bar above the traces indicates the duration of the retraction phase, based on large unit extracellular activity in BN2 (Morton and Chiel, 1993a,b; Church and Lloyd as a smaller-amplitude rejection-like motor pattern. A2, Effect of premature activation of the I1/I3/jaw complex on tube movement. A smaller-amplitude rejection-like motor pattern was induced using esophageal nerve stimulation. Just before the end of The tube moved back into the buccal cavity during this stimulation, so that the net outward movement of the tube was zero. In four experiments with no premature activation of BN2, the average outward tube movement during smaller-amplitude rejection-like movement was significantly reduced $(3.8 \pm 0.5 \mathrm{~mm} ; p<0.05 ; n=4)$. $\boldsymbol{B}$, Premature activation of the $11 / \mathrm{l} / \mathrm{j}$ jaw complex during pattern, as the tube both moved outward and began to rotate, the motor neurons for the $11 / 13 /$ jaw complex were stimulated by stimulating BN2 ( $20 \mathrm{~Hz}$ for $5 \mathrm{~s}$ ). The tube moved back into the buccal cavity, and the net outward movement was zero. In fou patterns was $8.3 \pm 1.3 \mathrm{~mm}$. In contrast, when the I1/l3/jaw complex was prematurely activated, the average outward tube movement was significantly reduced $(4.8 \pm 0.5 \mathrm{~mm} ; p<0.01 ; n=4)$.

window discriminator algorithm to classify units into "largest," "second largest," and "third largest units" (Morton and Chiel, 1993a; Ye et al., 2006), it is possible to obtain evidence for the activity of individual identified motor neurons in intact, behaving animals.

To minimize disruption to feeding responses, we generally implanted electrodes on one nerve at a time or on the I 2 muscle alone. We recorded individually from BN2 in six animals, from $\mathrm{BN} 3$ in eight animals, from $\mathrm{I} 2$ in seven animals, and from the RN in five animals. We were able to obtain simultaneous recordings from both $\mathrm{BN} 2$ and $\mathrm{BN} 3$ in two animals, $\mathrm{RN}$ and $\mathrm{BN} 2$ recordings in three animals, and $\mathrm{I} 2$ and BN2 recordings in two animals. Each animal performed at least five rejections.

We confirmed all three predictions by recordings in intact, 

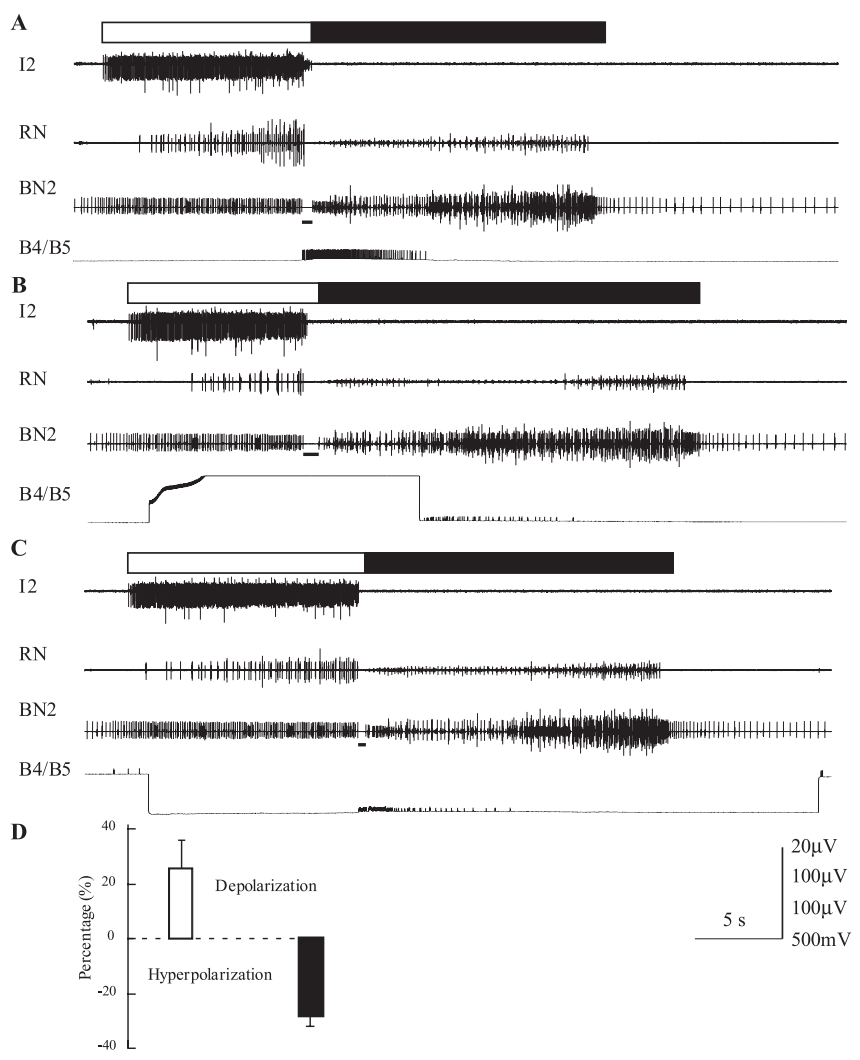

Figure 4. Changing the activity of a $B 4 / B 5$ neuron can alter the delay from the end of $\mathrm{I} 2$ activity to the onset of activity in the 11/13/jaw complex. $A$, Simultaneous extracellular recordings were obtained from the $\mathrm{I} 2$ nerve, $\mathrm{RN}$, and BN2 in an isolated buccal ganglion. A single B4/B5 neuron was impaled with an intracellular electrode. Note the much lower gain on the B4/B5 recording to ensure that activity in the neuron could still be monitored while it was strongly depolarized or hyperpolarized. Rejection-like patterns were evoked by stimulation of the esophageal nerve at $20 \mathrm{~Hz}$ for $2 \mathrm{~s}$. The open bar above the recordings indicates the protraction phase; the filled bar indicates the retraction phase. Large unit RN activity (corresponding to activity in the B8 motor neurons) (Morton and Chiel, 1993b) occurs during the protraction phase, the criterion for classifying a motor pattern as egestive (Morton and Chiel, 1993a,b; Church and Lloyd, 1994). A line under the BN2 record indicates the delay from the end of activity in the 12 motor neurons (i.e., the end of protraction) to the onset of activity in the $11 / 13 / \mathrm{jaw}$ complex motor neurons. Note that the $B 4 / B 5$ neuron becomes active just at the end of protraction and fires vigorously before the onset of activity in the $11 / 3 / 3$ jaw complex motor neurons (i.e., before large unit extracellular activity in BN2). B, Depolarizing a B4/B5 neuron delays the onset of activity in the I1/13/jaw complex motor neurons. Strong depolarizing current was injected into the $B 4 / B 5$ neuron at the onset of activity in the 12 nerve, inducing the $B 4 / B 5$ neuron to fire at a much higher rate. Note that the line under the BN2 trace representing the delay to onset of activity in the I1/13/jaw complex motor neurons is longer than the corresponding line in A. Note also the reduction in firing frequency of large units on RN, consistent with the effect of B4/B5 on the B8 motor neurons (Jing and Weiss, 2001). The change in RN activity does not affect the duration of the gap, which is measured from the offset of $\mathrm{I} 2$ activity to the onset of large unit activity in BN2. C, Hyperpolarizing a B4/B5 neuron reduces the delay to onset of activity in the I1/13/jaw complex motor neurons. Strong hyperpolarizing current was injected into the B4/B5 neuron at the onset of activity in the 12 nerve, inducing the neuron to fire at a much lower rate. Note that the line under the BN2 trace representing the delay to onset of activity in the I1/3/jaw complex motor neurons is shorter than the corresponding line in $A$. Data in $A-C$ are all from the same preparation. $\boldsymbol{D}$, Statistical summary of results. On average, depolarizing the $B 4 / B 5$ neuron increased the delay to onset of activity in the $11 / 3 /$ /jaw motor neurons by $26 \pm 10 \%$ relative to the delay in the immediately preceding motor pattern $(p<0.001 ; n=5)$. On average, hyperpolarizing the $B 4 / B 5$ neuron decreased the delay to onset of activity in the $11 / 13 / j a w$ motor neurons by $28 \pm 4 \%$ relative to the delay in the immediately preceding motor pattern $(p<0.001 ; n=4)$.

behaving animals (Fig. 6 shows data from individual animals, Fig. 7 shows averaged data for smaller-amplitude type A rejections, and Fig. 8 shows averaged data for larger-amplitude for type B swallows) ( $n=5$ animals for each average trace).
(1) The duration of I2 activity during type B rejections, in which tube ventral rotation was observed, was significantly longer than the duration of I2 activity during type A rejections (Figs. 6A, $B$, lines under traces labeled I2 EMG, 7A, 8A). The duration of I2 activity during type A rejections was $2.2 \pm 0.4 \mathrm{~s}$, whereas I2 duration was $3.0 \pm 0.4 \mathrm{~s}$ during type $\mathrm{B}$ rejections ( $p<$ $0.05 ; n=5)$.

(2) The B7 motor neuron (third largest unit on BN3) was highly active throughout rejection, so that it was active during the rotation phase of protraction during type $\mathrm{B}$ rejections. It continued to be active at the onset of the retraction phase in both type $A$ and type $B$ rejections, suggesting that the hinge muscle initiated retraction (Figs. 7C, $8 C$ ) (Ye et al., 2006).

(3) The onset of activity in the B10 motor neuron (third largest unit on BN2, which is the earliest motor neuron for the I1/I3/ jaw complex to become active) (Morton and Chiel, 1993a,b; Church and Lloyd, 1994) is delayed and is significantly more delayed in type B than in type A rejections (Fig. 6, note the relative lengths of the lines immediately beneath the BN2 traces that indicate the duration from the end of outward tube movement and the onset of $\mathrm{B} 10$ activity in type $\mathrm{A}$ and type $\mathrm{B}$ rejections; also note double-headed arrows in Figs. $7 \mathrm{H}, 8 \mathrm{H}$ ). Ratios of the delay from the time of onset of activity in the B10 unit after the tube stops moving out in type A and immediately succeeding type B rejections show that the delay is $33 \pm 22 \%$ greater in type B rejections $(n=5$ pairs from 2 animals; $p<0.05$; ratios were computed because of the high variability in the motor patterns) (Brezina et al., 2006).

(4) During type B rejections, B4/B5 activity (indicated by the largest extracellular unit on BN3) (Warman and Chiel, 1995) begins earlier and occurs at higher frequency during the delay from the end of outward tube movement to the onset of activity in the motor neurons for the I1/I3/jaw complex (note lines marked "B4/B5 onset" and timing of onset and duration of line underneath BN3 traces in Fig. 6 in type A vs type B rejections; also note activity in Figs. $7 E$ and $8 E$ before the dotted lines in $7 F-H$ and $8 F-H$.). The time of onset of activity in the B4/B5 unit once the tube begins moving out is $0.6 \pm 0.5 \mathrm{~s}$ in type A rejection, whereas it is $-1.2 \pm 0.4 \mathrm{~s}$ in type $\mathrm{B}$ rejections $(p<0.001$; the activity in $\mathrm{B} 4 / \mathrm{B} 5$ starts before the tube begins to move out in type $\mathrm{B}$ rejections) (Figs. 6, BN3 traces, $7 E, 8 E$ ). The duration of activity in the $\mathrm{B} 4 / \mathrm{B} 5$ unit is $2.6 \pm 0.6 \mathrm{~s}$ in type A rejection, whereas it is $5.6 \pm 0.6 \mathrm{~s}$ in type $\mathrm{B}$ rejections $(p<0.001)$. The maximum frequency in the B4/B5 unit is $28 \pm 4 \mathrm{~Hz}$ in type A rejection, whereas it is $45 \pm 7 \mathrm{~Hz}$ in type $\mathrm{B}$ rejections $(p<0.001)$. Finally, in vivo duration of $\mathrm{B} 4 / \mathrm{B} 5$ activity was significantly correlated with the amplitude of outward tube movement, as would be predicted if B4/B5 neuronal activity acted to delay onset of I1/I3/jaw activation and thus prolonged the protraction of rejection (the correlation coefficient $r$ between B4/B5 activity duration in seconds and the distance of outward tube movement in millimeters is 0.94; $p<0.001 ; n=16$ ).

Features 1 and 4 above, both consequences of the mechanical reconfiguration of the buccal mass during rejection, could be used to discriminate smaller-amplitude (type A) rejections from larger-amplitude (type B) rejections. Type B rejections were associated with significantly longer duration activity of the I2 muscle and with significantly earlier onset of activity in the B4/B5 neuron relative to the onset of outward movement of the tube. Distributions of the duration of I2 activity in type A and type B behaviors were constructed ( $n=5$ animals; 37 type A rejections, 19 type B rejections). A Kolmogorov-Smirnov test to compare the two distributions suggested that they were significantly dif- 
ferent from one another $(p<0.01)$ (Sokal and Rohlf, 1981). The duration of I2 activity that maximally discriminated between type A and type B swallows was $3.1 \pm 0.3 \mathrm{~s}$ (based on a discriminant function) (Sokal and Rohlf, 1981). Distributions of the time of onset of activity in the B4/B5 unit relative to the onset of outward tube movement were also constructed ( $n=4$ animals; 25 type $\mathrm{A}$ responses, 10 type $\mathrm{B}$ responses). Onset of the B4/B5 unit earlier than the onset of tube movement was represented using negative values, whereas onset of the B4/B5 unit after the onset of tube movement was represented using positive values. A Kolmogorov-Smirnov test to compare the two distributions suggested that they were significantly different from one another $(p<0.01)$. The time of relative onset that maximally discriminated between type $\mathrm{A}$ and type $\mathrm{B}$ swallows was $-0.30 \pm 0.25 \mathrm{~s}$ (based on a discriminant function) (Sokal and Rohlf, 1981).

The results obtained from in vivo studies strongly support each of the predictions made in the in vitro studies for the effects that mechanical reconfiguration attributable to the change in the central grasper shape would have throughout rejection.

\section{Mechanical reconfiguration and the neuromechanics of rejection}

The schematic diagram shown in Figure 9 summarizes how neural control interacts with mechanical reconfiguration throughout a rejection response.

(1) To grasp inedible material, the B8 motor neurons are activated at the onset of the rejection response, activating the I4 muscles, firmly closing the halves of the radula/odontophore around the tube (Fig. 9 , RB1, arrows in top view indicate direction of radular closure). As the radula/ odontophore closes, it assumes an elongated ellipsoidal shape (Fig. 9, compare bottom views of RB1 and RB2). The I2

protractor muscle wraps around the radula/odontophore, so that I 2 is stretched. As a consequence, I 2 has better mechanical advantage and can generate more force. This is the first example of mechanical reconfiguration.

(2) To push inedible material out of the buccal cavity, the motor neurons for I2 are activated shortly after the B8 motor neurons are activated. In a type B rejection, they are activated for a longer duration than during a type A rejection. Initially, the I2 protractor muscle pushes the radula/odontophore anteriorly, which moves the firmly grasped tube out of the buccal cavity (Fig. 9, RB2a, arrows in bottom view indicate direction of contraction of I2 and outward tube movement). In a type B rejection, as I2 continues to contract, the radula/odontophore rotates anteriorly about the hinge muscle, so that the tube undergoes a ventral rotation (RB2b, arrows in the bottom view indicate the rotation about the hinge muscle and the ventral rotation of the tube). In a type A rejection, the protraction is not as large, and so the central grasper does not rotate about the hinge muscle.

(3) To release material so that it remains outside of the buccal cavity, the halves of the radula/odontophore open and release the tube (Fig. 9, RB3, arrows in top view indicate direction of radular opening). The I1/I3/jaw complex must not be activated or it will antagonize the opening of the radular halves and prevent tube release. Instead, motor neuron $\mathrm{B} 7$ activates the stretched hinge muscle to initiate retraction (Fig. 9, RB3, arrows in bottom view indicate direction of radular/odontophore rotation about the hinge muscle).

(4) To complete the retraction phase, motor neurons (e.g., B10) for the I1/I3/jaw complex are activated, inducing the radula/odontophore to move posteriorly back to its initial position (Fig. 9, RB4, arrows in the top view indicate the direction of contraction of the I1/I3 muscle and the resulting 


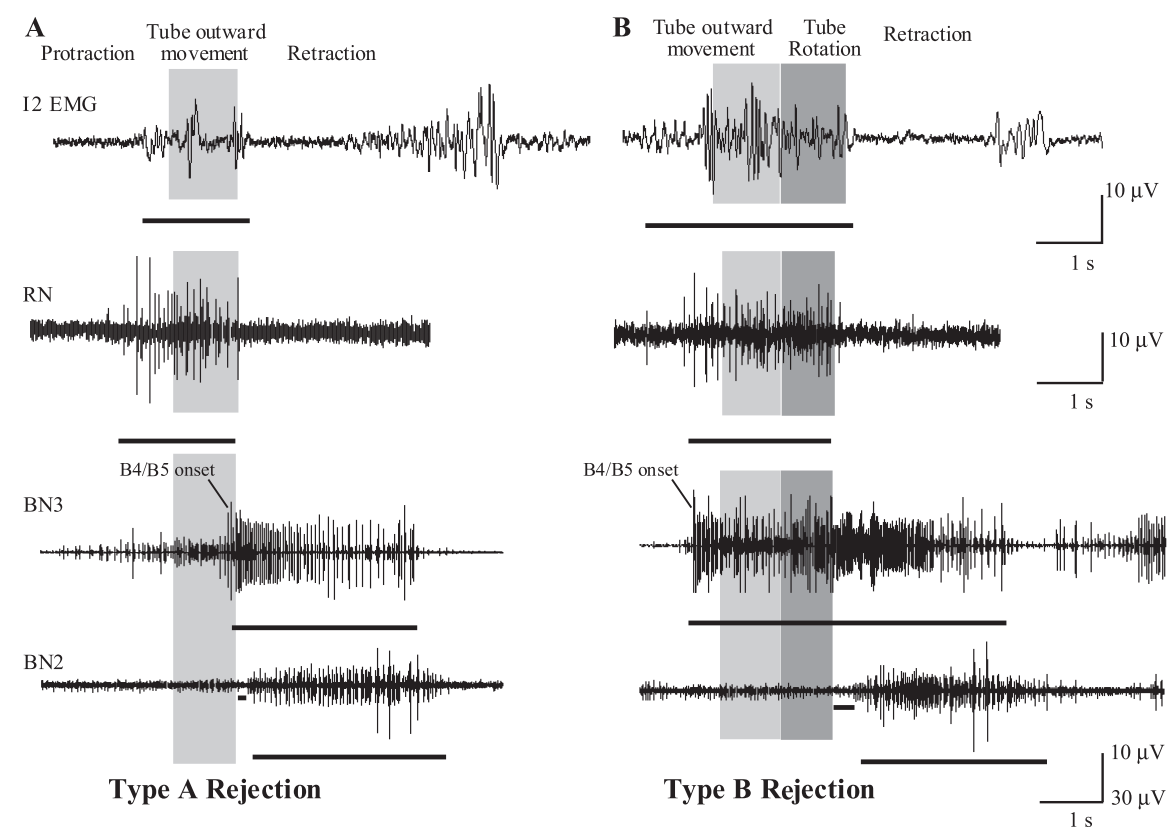

Figure 6. In vivo neural and muscular recordings from intact, behaving animals during type A and type B rejections. Bars underneath traces in both parts of the figure indicate large unit activity identified based on a window discriminator algorithm (Morton and Chiel, 1993a; Ye at al., 2006). A, Activity on muscles and nerves during a type A rejection. Traces are from three different animals and are aligned by the onset of the outward translation of a tube, which is indicated using the shaded gray rectangle. Note that the large burst on the 12 EMG that occurs during the retraction phase (after the tube has moved outward) is likely to reflect activity on the underlying 14 muscle because it is larger in amplitude, lower in frequency, facilitates, and is not abolished by lesions of the $\mathrm{I} 2$ nerve, and is therefore not analyzed (Hurwitz et al., 1996, their Figs. 11 B, 14). $\boldsymbol{B}$, Activity on muscles and nerves during a type $B$ rejection. Traces from three different animals are aligned on the border between the outward translation and ventral rotation of the tube, which is indicated using a light or dark gray rectangle, respectively. Compared with the data shown in $\boldsymbol{A}$, there is an increase in the duration and frequency of the $12 \mathrm{EMG}$, an increase in B4/B5 activity on BN3 (i.e., in the activity of the largest extracellular unit), and an increase in the delay before the third largest BN2 unit begins.

movement of the radula/odontophore; bottom view shows movement of the radula/odontophore, which has released the tube; final configuration is shown in the bottom right panels, labeled RB1; note that the mark on the tube has moved out of the buccal cavity).

In summary, mechanical reconfiguration is important for the control of rejection in Aplysia. In particular, the ellipsoidal shape of the central grasper increases the length of the 12 muscle, and, as predicted by a kinetic model (Novakovic et al., 2006), the resulting increase in the mechanical advantage of $\mathrm{I} 2$ and its ability to generate force allow it to generate strong protractions (Fig. 1). As a consequence of the strong I2 protractions and the closed configuration of the grasper, the hinge muscle is now involved in a new function, generating ventral rotations during the protraction phase (Figs. 2, 5). After the grasper opens to release inedible food, its new shape imposes a constraint on the activation of the surrounding I1/I3/jaw muscles, which will close the grasper if activated prematurely and interfere with rejection (Fig. 3). Activity in the B4/B5 neurons appears to mediate this delay in the onset of I1/I3/jaw complex activity (Fig. 4). All of the in vitro predictions for the effects of mechanical reconfiguration are reflected in the in vivo neural and muscular recordings (Figs. 6-8) and provide a much deeper explanation for the interactions of neural control and mechanics during rejection (Fig. 9).

\section{Discussion}

\section{Mechanical reconfiguration and} neuromechanical modulation

Mechanical reconfiguration may affect neural control whenever changes in muscle shape affect the shapes or positions of other muscles. In other mollusks, shape changes of the radula/odontophore during feeding may alter the mechanics of surrounding muscles (Rose, 1971; Rose and Benjamin, 1979; Smith, 1988; Messenger and Young, 1999). For example, in the buccal masses of Octopus bimaculoides, Sepia officinallis, and Loliguncula brevis, if the lateral mandibular muscles are relaxed, the posterior mandibular muscle closes the beak. If the lateral mandibular muscles are tensed, the posterior mandibular muscle opens the beak, a form of mechanical reconfiguration termed "muscular articulation" (Uyeno and Kier, 2005).

Mechanical reconfiguration is also important in vertebrates. For example, if a snake's body is straight, phasic contractions of epaxial muscles induce swallowing. In contrast, if its body is curved, phasic contractions of the epaxial muscles induce locomotion (Moon, 2000).

Because of mechanical reconfiguration, similar durations of motor neuron activation can lead to different amplitude outputs. Although the duration of I2 activation in type $B$ swallows is similar to the duration of $\mathrm{I} 2$ activation in type A rejections $(2.4 \pm 0.6$ vs $2.2 \pm 0.4 \mathrm{~s}$ ) (Fig. $7 \mathrm{~A}$ ) (Ye et al., 2006), protractions associated with type $B$ swallows are not as large as those associated with type A rejections, as judged by the number of times the radular surface is visible $[15 \%$ (6 of 39 ) times in type B swallows vs 35\% (20 of 57) type A rejections; $\chi^{2}$ statistic of 4.552; $p<0.033$ ]. Thus, the effectiveness of the motor neurons for $\mathrm{I} 2$ as protractors (B31/B32, B61/B62) is altered by activity in a different set of motor neurons, the B8 radular closers.

Mechanical reconfiguration is an example of a broader form of modulation. When one neuron has no direct effect on another neuron or a muscle but alters the strength of the output of the second neuron or the muscle, this interaction is termed "neuromodulation" (LeBeau et al., 2005). A similar phenomenon of "neuromechanical modulation" may occur via the periphery: one neuron does not directly induce motor output from a muscle but indirectly affects the output of other motor neurons through mechanical coupling. Given the inertial coupling between distant joints during rapid movements, as well as the role of postural muscles in allowing other muscles to generate movement (Zajac, 1993; Balasubramaniam and Wing, 2002), it is likely that neuromechanical modulation is widespread and behaviorally significant.

\section{Variants and variability}

Are type B swallows or rejections different variants of swallowing or rejection, or simply larger-amplitude versions of type A swallows or rejections? In other words, are they similar to distinct "gaits" of locomotion that vary in speed (e.g., pace, canter, trot, or gallop), or are they just stronger versions of the same behavior? Behavioral distinctions between gaits are based on movement patterns. Type A and B swallows are distinct because tube movements are different from the onset. In contrast, type A and B rejections are initially identical (Fig. 5D), but large ventral rota- 


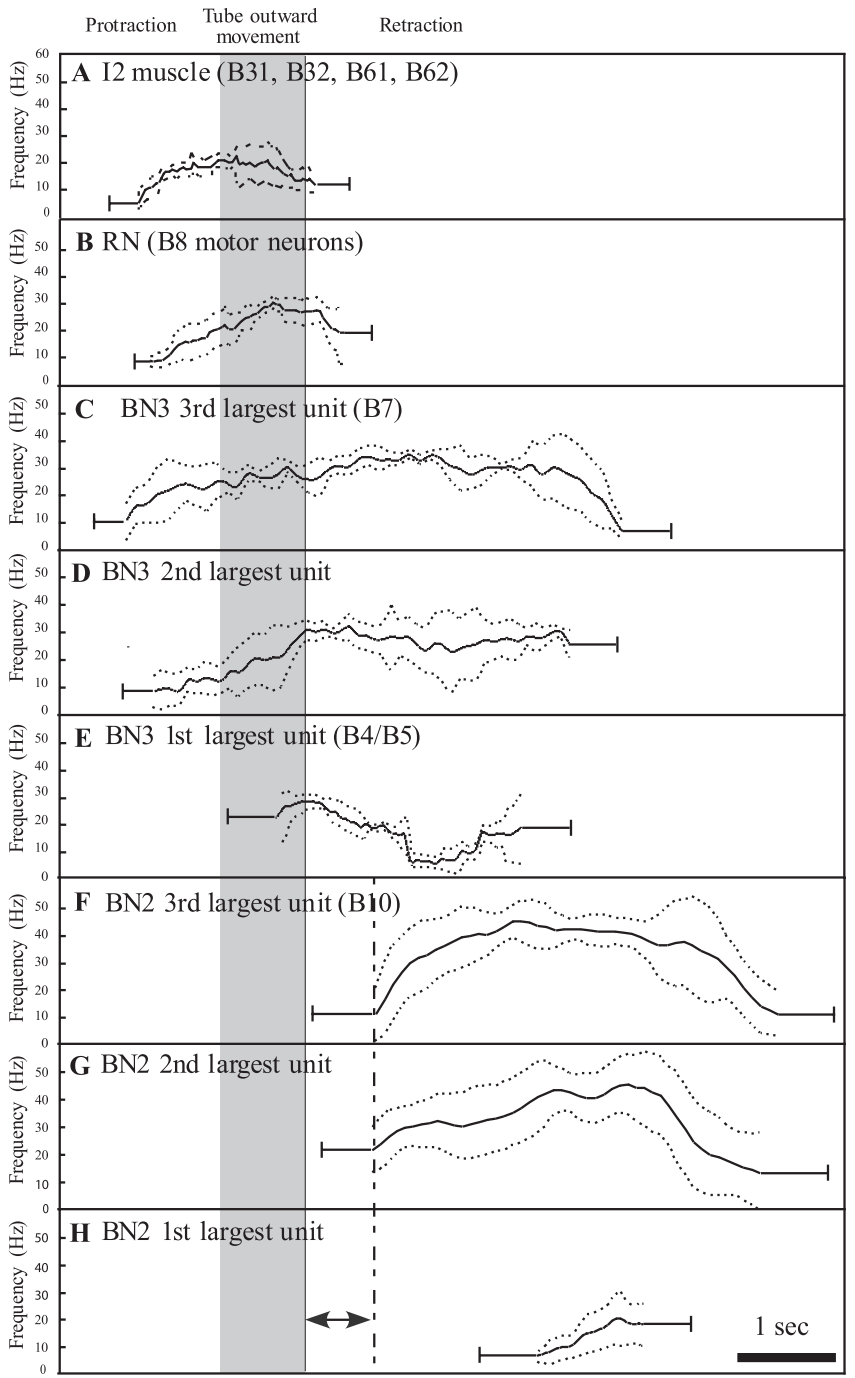

Figure 7. Statistical summary of nerve and muscle activity during type A rejections. Neural and muscular activity from recordings of single nerves or the $\mathrm{I} 2$ muscle in freely behaving animals ( $n=5$ for each trace) were synchronized with the outward translation of the tube, and their durations were normalized by the duration of the outward movement to create a composite summary. Three different frequency profiles were obtained from BN2 and BN3, respectively, corresponding to motor units of different sizes (Morton and Chiel, 1993a,b; Ye et al., 2006, their Materials and Methods). One frequency profile was generated for RN activity. Neural recordings from multiple type A swallowing responses were averaged (solid curves), and their SDs were calculated and plotted (dotted lines). Before and after the solid central line in each panel, the mean \pm SD of the onset and offset time are shown using solid lines. Arrow in $\boldsymbol{H}$ indicates the delay from the end of protraction to the onset of activity in units innervating the I1/l3/jaw complex on BN2.

tions after outward translation unequivocally distinguish type B rejections. Type B rejections are also distinguished neurally by early activity in the B4/B5 neurons (Figs. 6-8).

Our data suggest that higher rejection intensity results from neural changes that can be measured in vitro: longer $\mathrm{I} 2$ activation, and early onset and higher intensity of B4/B5 activity. Reports that $\mathrm{B} 65$ activity or dopamine reduces $\mathrm{I} 2$ activation duration during rejection-like patterns in isolated ganglia (Due et al., 2004) suggest that dopamine decreases rejection intensity. Due et al. reached the opposite conclusion by focusing on the firing intensity of the B8 motor neuron. Analyzing the effects of dopamine in a semiintact preparation will resolve these differing interpretations.

Behavioral variants can give rise to variability in behavior. For example, if animals generate different types of swallows or rejec-

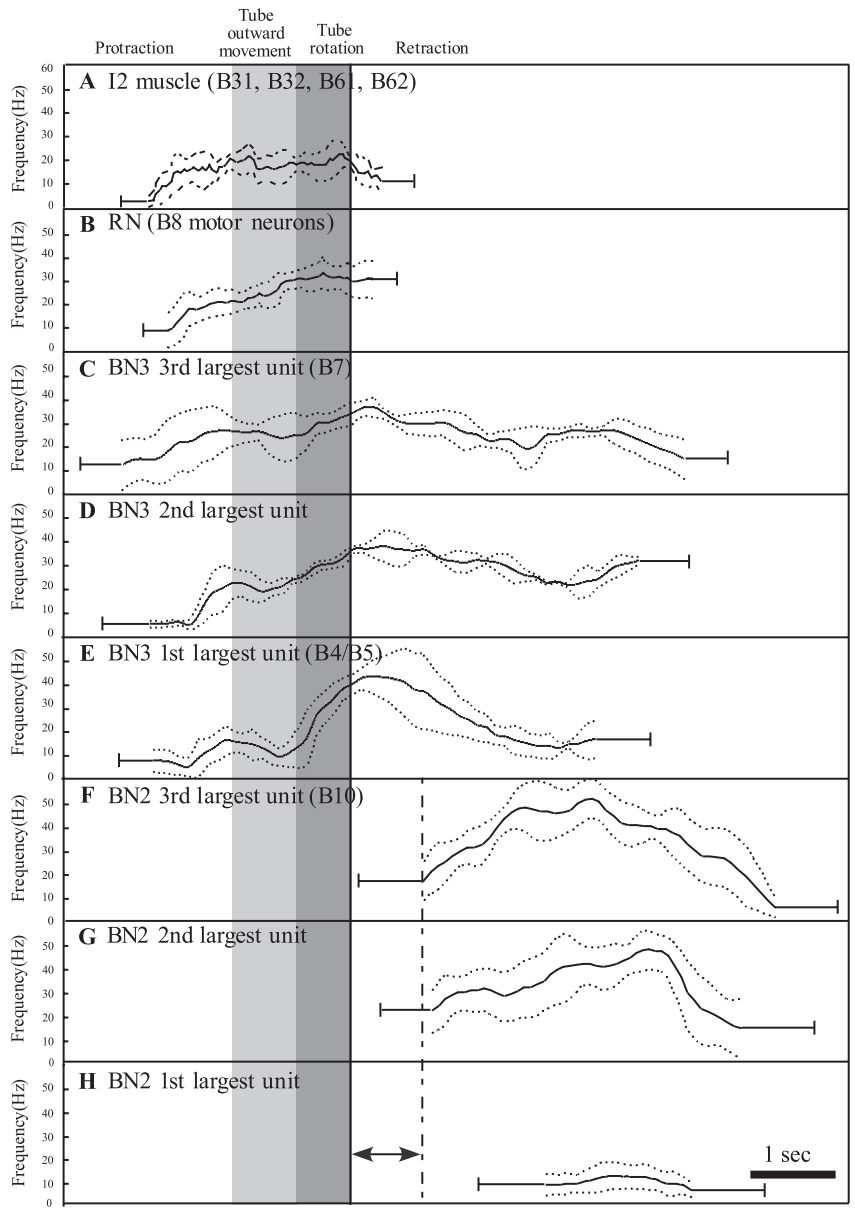

Figure 8. Statistical summary of nerve and muscle activity during type B rejections. Light gray bar indicates timing of tube outward movement; dark gray bar indicates timing of tube ventral rotation. For details, see legend to Figure 7 .

tions in response to soft materials, their responses and neural activity will have higher variability, because variants having smaller variability cannot be distinguished. The responses of Aplysia to uniform tubes results also emphasize the importance of sensory input for variability. The tube does not change during swallowing or rejection, unlike a seaweed strip that may bunch up as it is consumed, and yet animals generate different responses. This may be attributable to different sensory inputs as the tube contacts parts of the radula, pharyngeal tissue, esophagus, and anterior crop, or it may be attributable to inherent variability in the pattern generator. Intrinsic variability may be important to allow animals to rapidly adjust feeding movements to changing loads from seaweed (Brezina et al., 2006). Controlling sensory input will be essential for clarifying these two possibilities.

\section{Multifunctionality and muscular coalitions}

Although muscles are often described by a single function, this may be a misleading oversimplification. Muscles may form coalitions to perform behavioral functions. During type B swallows, retraction is mediated by a coalition of the I4 muscle, hinge muscle, and I1/I3/jaw complex (Fig. $10 \mathrm{~B}$ ). During type B rejections, protraction is mediated by a coalition of the $\mathrm{I} 2$ and the hinge muscles (Fig. 10D). In all rejections, retraction is attributable to a coalition of the hinge muscle and the I1/I3/jaw complex (Fig. $10 C, D)$. Under some circumstances, coalitions reduce to a single muscle (e.g., the I1/I3/jaw complex during retraction in type A 


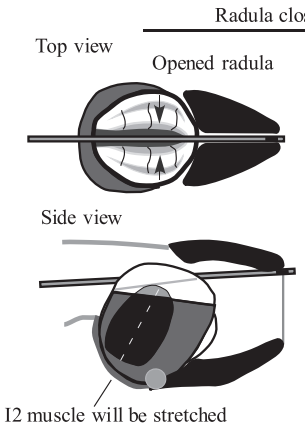

RB1
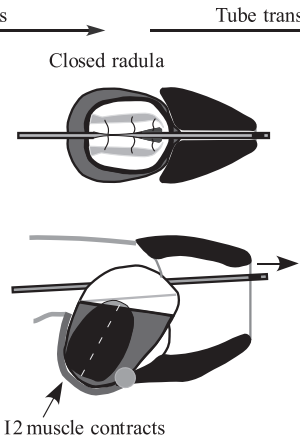

RB2a $\longrightarrow$
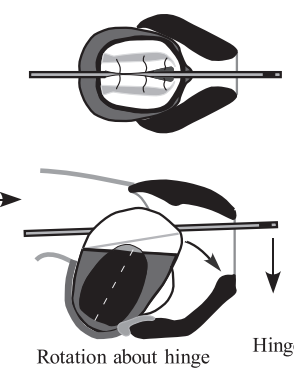

$\mathrm{RB} 2 \mathrm{~b}$
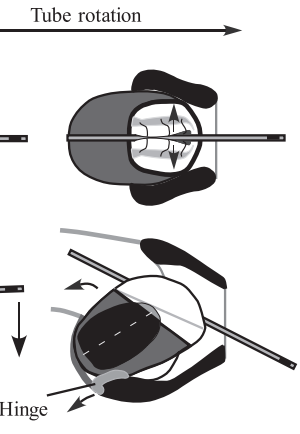

RB3

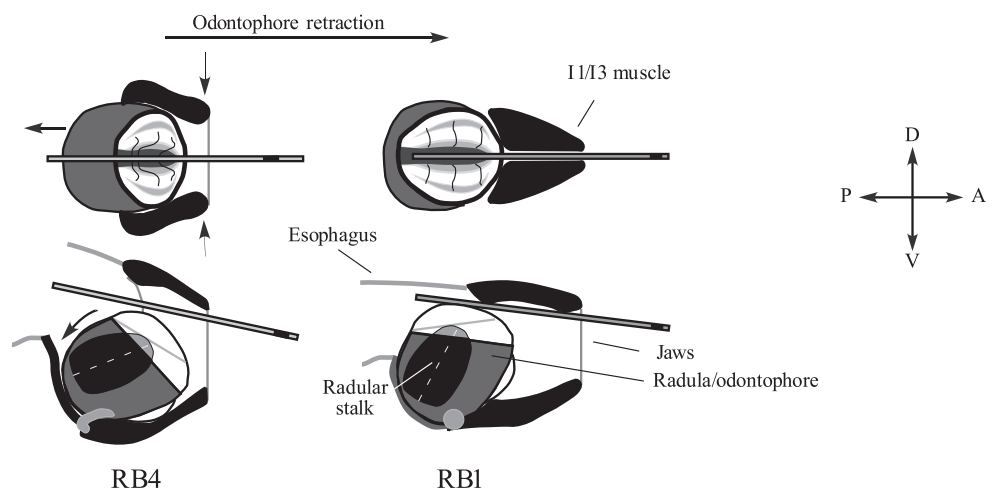

Figure 9. Schematic top and side views of movements of the muscles of the buccal mass during larger-amplitude (type B) rejections. For additional details, see Results. A, Anterior; D, dorsal; P, posterior; $V$, ventral.
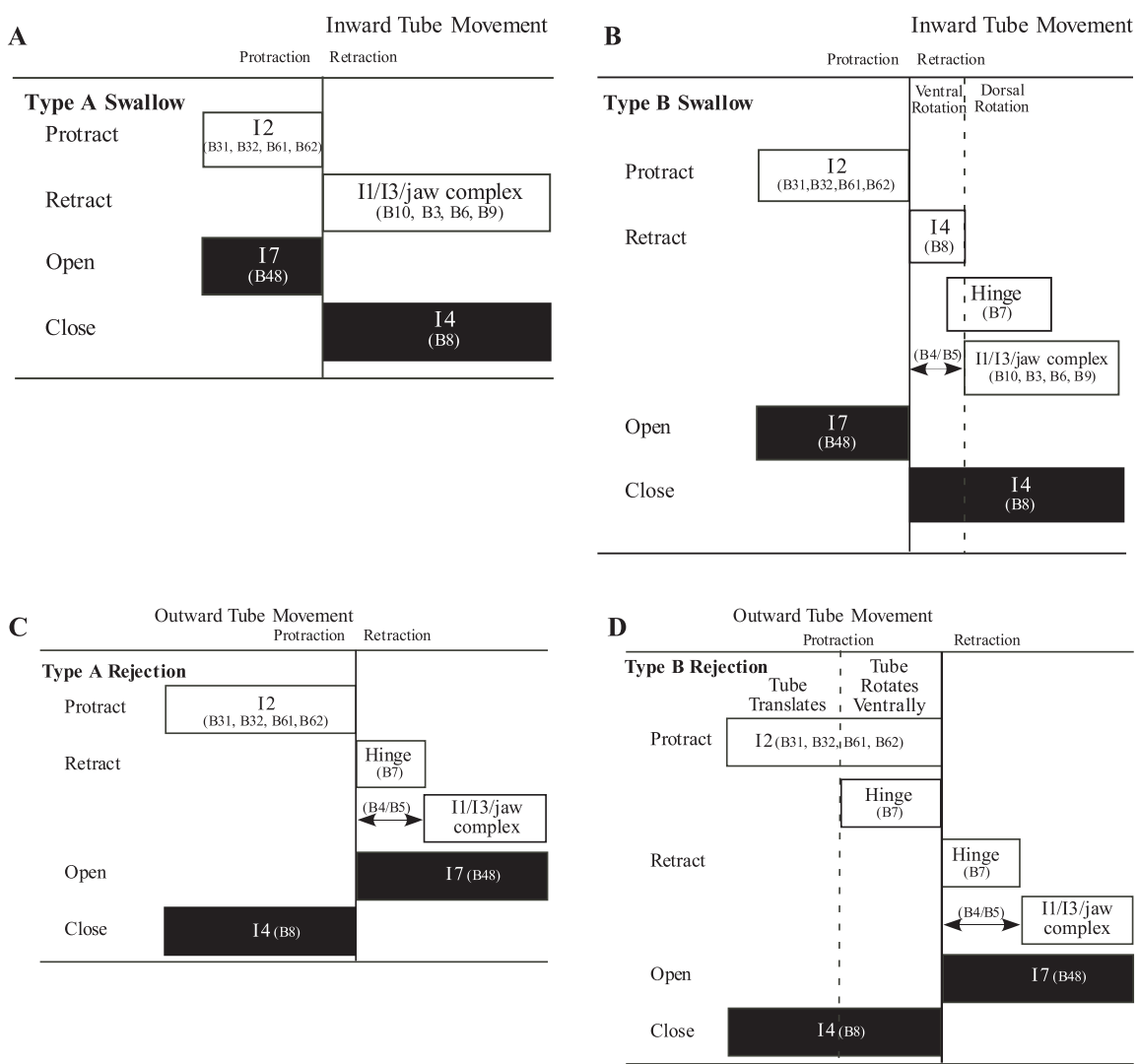

Figure 10. Schematic comparison of muscle functions in type A and type B swallows and rejections. For additional details, see Discussion. swallows) (Fig. 10A), so in that context it is correct to refer to the muscle as mediating that function, but this may vary with context. For example, the I4 muscle acts as a closer muscle in type A swallows and in type $\mathrm{A}$ and type $\mathrm{B}$ rejections, but $\mathrm{I} 4$ has both closer and retractor functions during type B swallows (Fig. 10B). A muscle should only be assigned a single functional role if, in all behavioral contexts, it always plays a single role. These observations parallel results that have been found at the motor neuronal level, in which motor neurons in the stomatogastric nervous system may dynamically form different coalitions and participate in very different motor patterns (Weimann et al., 1991).

Muscular coalitions may underlie behavioral flexibility. If each muscle has only one function, a system is more limited than one in which many muscular subsets may be used for different functions. Shifting muscle coalitions are likely to apply to vertebrate systems as well, as illustrated by multiarticular anatomy (Lombard and Abbott, 1907; Kargo and Rome, 2002) and the changing roles of muscles in detailed simulations of bicycling and locomotion (Zajac et al., 2002, 2003). Combinatorial structures that enhance the flexibility of biological systems are observed in protein synthesis (Le Hir et al., 2003), development (Louvi and Artavanis-Tsakonas, 2006), and the nervous system (Jing and Weiss, 2001).

\section{Implications for neural control of multifunctionality}

Muscular coalitions are deployed for different behaviors by varying the duration, phasing, and intensity of motor neuronal activation. A major difference between type $A$ and $B$ swallows and between type $A$ and $B$ rejections is the duration of $I 2$ activation (Figs. 6, I2 EMG traces, 7A, 8A) (Ye et al., 2006, their Figs. 10-12). Changes in the phases of activity in the radular closer motor neurons B8a/b distinguish ingestion from rejection (Figs. 6, RN traces, $7 B$, 8B) (Morton and Chiel, 1993a,b; Ye et al., 2006, their Figs. 10-12). Type B rejections also differ from type $\mathrm{A}$ in the earlier onset (earlier phase), longer duration, and higher intensity of firing in the B4/B5 neurons (Figs. 6, BN3 traces, $7 E, 8 E$ ).

Changes in duration, phasing, and intensity of motor neuronal activity are seen in other multifunctional systems. Such changes are seen in the shift from forward to backward locomotion in salamander (Ashley-Ross and Lauder, 1997) or bicycle pedaling in humans (Neptune et al., 2000), in turtles as they shift from walking to 
swimming (Stein, 2005), and in eels, toads, turtles, and rats as they shift from walking on land to walking through water (Gillis and Blob, 2001).

Peripheral biomechanics determine the behavioral significance of changes in duration, phasing, and intensity. For example, the gap after $\mathrm{I} 2$ activity to activity in the I1/I3/jaw complex motor neurons reflects the need to delay its contraction to allow larger protractions. Quiescence in the neural record may therefore indicate that behaviorally relevant dynamics has shifted to the periphery. Furthermore, a small shift in phasing at the neural level, played through the biomechanics of the body, leads to a much larger shift in behavior, a form of "differential penetrance."

Studies using in vitro patterns demonstrated that shifts in the phase at which the grasper closes, controlled by shifts in timing of B8 motor neuron activity, may be attributable to the actions of interneurons CBI-2 and CBI-3 (Jing and Weiss, 2001) acting through interneurons B20 and B40 (Jing and Weiss, 2002). Control of $\mathrm{I} 2$ duration and intensity may be attributable to actions of GABAergic interneurons (Jing et al., 2003). It is clear that the combination of shifting coalitions of muscles, and a reorganizing neural architecture, are critical for multifunctionality. These observations support the hypothesis that behavioral control can best be understood within a biomechanical context (Chiel and Beer, 1997; Dickinson et al., 2000).

\section{References}

Ashley-Ross MA, Lauder GV (1997) Motor patterns and kinematics during backward walking in the Pacific Giant Salamander: evidence for novel motor output. J Neurophysiol 78:3047-3060.

Balasubramaniam R, Wing AM (2002) The dynamics of standing balance. Trends Cogn Sci 6:531-536.

Berkowitz A (2001) Broadly tuned spinal neurons for each form of fictive scratching in spinal turtles. J Neurophysiol 86:1017-1025.

Berkowitz A (2002) Both shared and specialized spinal circuitry for scratching and swimming in turtles. J Comp Physiol A Neuroethol Sens Neural Behav Physiol 188:225-234.

Brezina V, Proekt A, Weiss KR (2006) Cycle-to-cycle variability as an optimal behavioral strategy. Neurocomputing 69:1120-1124.

Buneo CA, Soechting JF, Flanders M (1997) Postural dependence of muscle actions: implications for neural control. J Neurosci 17:2128-2142.

Chiel HJ, Beer RD (1997) The brain has a body: adaptive behavior emerges from interactions of nervous system, body and environment. Trends Neurosci 20:553-557.

Chiel HJ, Weiss KR, Kupfermann I (1986) An identified histaminergic neuron modulates feeding motor circuitry in Aplysia. J Neurosci 6:2427-2450.

Church PJ, Lloyd PE (1994) Activity of multiple identified motor neurons recorded intracellularly during feedinglike motor programs in Aplysia. J Neurophysiol 72:1794-1809.

Dickinson MH, Farley CT, Full RJ, Koehl MA, Kram R, Lehman S (2000) How animals move: an integrative view. Science 288:100-106.

Due MR, Jing J, Weiss KR (2004) Dopaminergic contributions to modulatory functions of a dual-transmitter interneuron in Aplysia. Neurosci Lett 358:53-57.

Gardner D (1971a) Synaptic organization and bilateral symmetry in the buccal ganglia of Aplysia. PhD thesis, New York University.

Gardner D (1971b) Bilateral symmetry and interneuronal organization in the buccal ganglia of Aplysia. Science 173:550-553.

Gillis GB, Blob RW (2001) How muscles accommodate movement in different physical environments: aquatic vs. terrestrial locomotion in vertebrates. Comp Biochem Physiol A Mol Integr Physiol 131:61-75.

Harris-Warrick RM, Marder E, Selverston AI, Moulins M (1992) Dynamic biological networks: the stomatogastric nervous system. Cambridge, MA: MIT.

Hurwitz I, Neustadter D, Morton DW, Chiel HJ, Susswein AJ (1996) Activity patterns of the $\mathrm{B} 31 / \mathrm{B} 32$ pattern initiators innervating the $\mathrm{I} 2$ muscle of the buccal mass during normal feeding movements in Aplysia californica. J Neurophysiol 75:1309-1326.
Jing J, Weiss KR (2001) Neural mechanisms of motor program switching in Aplysia. J Neurosci 21:7349-7362.

Jing J, Weiss KR (2002) Interneuronal basis of the generation of related but distinct motor programs in Aplysia: implications for current neuronal models of vertebrate intralimb coordination. J Neurosci 22:6228-6238.

Jing J, Vilim FS, Wu J-S, Park J-H, Weiss KR (2003) Concerted GABAergic actions of Aplysia feeding interneurons in motor program specification. J Neurosci 23:5283-5294.

Kargo WJ, Rome LC (2002) Functional morphology of proximal hindlimb muscles in the frog Rana pipiens. J Exp Biol 205:1987-2004.

Katzoff A, Ben-Gedalya T, Hurwitz I, Miller N, Susswein YZ, Susswein AJ (2006) Nitric oxide signals that Aplysia have attempted to eat, a necessary component of memory formation after learning that food in inedible. J Neurophysiol 96:1247-1257.

Kupfermann I (1974) Feeding behavior in Aplysia: a simple system for the study of motivation. Behav Biol 10:1-26.

Le Hir H, Nott A, Moore MJ (2003) How introns influence and enhance eukaryotic gene expression. Trends Biochem Sci 28:215-220.

LeBeau FE, El Manira A, Grillner S (2005) Tuning the network: modulation of neuronal microcircuits in the spinal cord and hippocampus. Trends Neurosci 28:552-561.

Lombard WP, Abbott FM (1907) The mechanical effects produced by the contraction of individual muscles of the thigh of the frog. Am J Physiol 20:1-60.

Louvi A, Artavanis-Tsakonas S (2006) Notch signaling in vertebrate neural development. Nat Rev Neurosci 7:93-102.

Messenger JB, Young JZ (1999) The radular apparatus of cephalopods. Philos Trans R Soc Lond B Biol Sci 354:161-182.

Moon BR (2000) The mechanics of swallowing and the muscular control of diverse behaviours in gopher snakes. J Exp Biol 203:2589-2601.

Morton DW, Chiel HJ (1993a) In vivo buccal nerve activity that distinguishes ingestion from rejection can be used to predict behavioral transitions in Aplysia. J Comp Physiol A Neuroethol Sens Neural Behav Physiol 172:17-32.

Morton DW, Chiel HJ (1993b) The timing of activity in motor neurons that produce radula movements distinguishes ingestion from rejection in Aplysia. J Comp Physiol A Neuroethol Sens Neural Behav Physiol 173:519-536.

Neptune RR, Kautz SA, Zajac FE (2000) Muscle contributions to specific biomechanical functions do not change in forward versus backward pedaling. J Biomech 33:155-164.

Neustadter DM, Drushel RF, Chiel HJ (2002a) Kinematics of the buccal mass during swallowing based on magnetic resonance imaging in intact, behaving Aplysia californica. J Exp Biol 205:939-958.

Neustadter DM, Drushel RF, Chiel HJ (2002b) A kinematic model of swallowing in Aplysia californica based on radula/odontophore kinematics and in vivo magnetic resonance images. J Exp Biol 205:3177-3206.

Novakovic VA, Sutton GP, Neustadter DM, Beer RD, Chiel HJ (2006) Mechanical reconfiguration mediates swallowing and rejection in Aplysia californica. J Comp Physiol A Neuroethol Sens Neural Behav Physiol 192:857-870.

Robertson GA, Stein PSG (1988) Synaptic control of hindlimb motonneurons during 3 forms of the fictive search reflex in the turtle. J Physiol (Lond) 404:101-128.

Rose RM (1971) Functional morphology of the buccal mass of the nudibranch Archidoris pseudoargus. J Zool Lond 165:317-336.

Rose RM, Benjamin PR (1979) The relationship of the central motor pattern to the feeding cycle of Lymnaea stagnalis. J Exp Biol 80:137-163.

Scott ML, Govind CK, Kirk MD (1991) Neuromuscular organization of the buccal system in Aplysia californica. J Comp Neurol 311:1-16.

Smith DA (1988) Radular kinetics during grazing in Helisoma trivolvis (Gastropoda: pulmonata). J Exp Biol 136:89-102.

Soffe SR (1991) Triggering and gating of motor-responses by sensory stimulation: behavioral selection in Xenopus embryos. Proc Biol Sci 246:197-203.

Sokal RR, Rohlf FJ (1981) Biometry. San Francisco: Freeman.

Stein P (2005) Neuronal control of turtle hindlimb motor rhythms. J Comp Physiol A Neuroethol Sens Neural Behav Physiol 191:213-229.

Sutton GP, Macknin JB, Gartman SS, Sunny GP, Beer RD, Crago PE, Neustadter DM, Chiel HJ (2004a) Passive hinge forces in the feeding apparatus of Aplysia aid retraction during biting but not during swallowing. J Comp Physiol A Neuroethol Sens Neural Behav Physiol 190:501-514. 
Sutton GP, Mangan EV, Neustadter DM, Beer RD, Crago PE, Chiel HJ (2004b) Neural control exploits changing mechanical advantage and context dependence to generate different feeding responses in Aplysia. Biol Cybern 91:333-345.

Uyeno TA, Kier WM (2005) Functional morphology of the cephalopod buccal mass: a novel joint type. J Morphol 264:211-222.

Warman EN, Chiel HJ (1995) A new technique for chronic single-unit extracellular recording in freely behaving animals using pipette electrodes. J Neurosci Methods 57:161-169.

Weimann JM, Marder E (1994) Switching neurons are integral members of multiple oscillatory networks. Curr Biol 4:896-902.

Weimann JM, Meyrand P, Marder E (1991) Neurons that form multiple pattern generators: identification and multiple activity patterns of gastric pyloric neurons in the crab stomatogastric system. J Neurophysiol 65:111-122.

Weiss EJ, Flanders M (2004) Muscular and postural synergies of the human hand. J Neurophysiol 92:523-535.
Weiss KR, Chiel HJ, Koch U, Kupfermann (1986) Activity of an identified histaminergic neuron, and its possible role in arousal of feeding behavior in semi-intact Aplysia. J Neurosci 6:2403-2415.

Ye H, Morton DW, Chiel HJ (2006) Neuromechancis of coordination during swallowing in Aplysia californica. J Neurosci 26:1470-1485.

Yu SN, Crago PE, Chiel HJ (1999) Biomechanical properties and a kinetic simulation model of the smooth muscle I2 in the buccal mass of Aplysia. Biol Cybern 81:505-513.

Zajac FE (1993) Muscle coordination of movement: a perspective. J Biomech 26:109-124.

Zajac FE, Neptune RR, Kautz SA (2002) Biomechanics and muscle coordination of human walking. I. Introduction to concepts, power transfer, dynamics and simulations. Gait Posture 16:215-232.

Zajac FE, Neptune RR, Kautz SA (2003) Biomechanics and muscle coordination of human walking. II. Lessons from dynamical simulations and clinical implications. Gait Posture 17:1-17. 